\title{
A FUZZY CELL-MAPPING FEEDBACK CONTROL ALGORITHM FOR THE SATELLITE ATTITUDE MANEUVERING CONTROL
}

\author{
Jia-Yush Yen \\ Associate Professor \\ Shou-Wen Tamg \\ Graduate Student \\ Department of Mechanical Engineering. \\ Vational Taiwan Linversit, Taipei. Taiwan, $1076+$ R.O.C.
}

\begin{abstract}
A fuzzy cell-mapping control algorithm formally proposed for motor control is extended to the application of satellite attitude maneuvering stabilizing control. The cell-mapping method treats the complex satellite attitude dynamics, and the fuzzy interpretation helps achieve a suitable control effort. The result is a smooth transition from manewvering to stabilization without overshoot while maintaining an optimal control performance.
\end{abstract}

\section{Introduction}

Many satellites are designed to point toward the earth. Some are intended to face certain objects in the space. To maintain these goals, the attitude control system must be an important entity in the satellite design (Hughes. 1986). In this paper, the fuzzy cell mapping method (Yen et. al., 1994) is extended to resolve the complex dynamic behavior in the satellite attitude maneuver control problem.

The satellite attitude is affected by many factors. First of all. the satellite attitude must change with the relative position in its orbit. Some spacecraft are large enough so that structural vibrations can not be neglected. The dynamics of fluids in the fiel tank can also affect the spacecraft attitude. Most of all, the dynamic equations that describe the satellite dynamics are highly non-linear. For the attitude stability. most of these effects are treated as small disturbances. The orbital interface is usually included in the dynamic model and treated as a varying reference set point. From the control point of view. the mathematics describing the evolving of the spacecraft orientation usually requires complicated coordinate transformations (Hughes. 1986. Bois and Kovalevsky. 1990). Further, the spacecraft dynamic characteristics is dependent on its rotational motion. To achieve the satellite attitude control, the control system generally divide the task into two separate processes: an attitude manewer control and an attitude stabilization control (Wertz. 1978). The attitude maneuver control is to move the satellite across large angular displacements to acquire certain pointing direction. The control algorithm is always based on rigid body dynamics so that complex rotational motion can be resolved. The attitude stabilization control is to maintain the satellite at a constant pointing direction. The control algorithm is often based on linearized dynamic model. Therefore. many advanced linear control theories have been employed (Dougherty, et. al. 1971. Wie. et. al. 1985). Most of the recent works 
have demonstrated successful applications on the large flexible space structures (Hale. et. al.: 1985, Byun and Wie, 1991). Very little result concerning the attitude manewvering control are found in the litcrature. The attitude maneuvering process undergoes large angular displacement. The control algorithm can not rely on the simple linearized model. Conventional satellite neglects the rotational effects and use a bang-bang or bang-zero-bang type control action to steer the satellite to a close proximity of the target attitude. The stabilization control system will then take over to regulate the satellite attitude around the reference point. Because energy is a major concern in the spacecraft operation, the maneuvering process can take months (Wertz, 1978).

In this paper, the fuzzy cell-mapping concept (Yen et. al,, 1994) is extended to the application of satellite attitude maneurer control. The satellite attitude dynamic model is based on Newton's second law. The cell-mapping analysis (Hsu, 1980, $1985,1987)$ takes into account all the rotational effects during the maneuvering process and searches for the optimal control sequence. The small control torque available can also be included in the consideration. The complex satellite attitude control problem would require a very large amount of cells to properly describe the attitude behavior. With the fuzzy cell-mapping interpretation, maneuvering control to position state is accomplished even though very large cell sizes are used.

\section{The Satellite Dynamic Model}

Consider body fixed coordinate which passes through the satellite mass center and is coincide with the principal axis. Because the period of the satellite going around the orbit is very slow compared with the attitude dynamics, the orbit coordinate is treated as an inertia coordinate. For simplicity, the authors use reaction wheels as control actuators. Consider three reaction wheels on three axes, the schematics of the satellite is represented as the following figure.

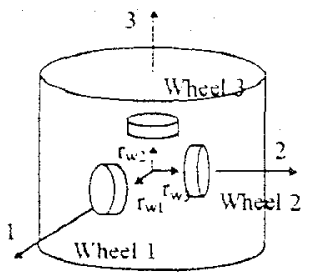

Figure 1 Satellite Control System Schematics

The angular momentum of the satellite can be described as (Hughes, 1986).

$$
\mathbf{H}^{S / C}=\mathbf{H}^{B C}+\sum_{i=1}^{3} \mathbf{H}^{B \cdot C} \text {. }
$$

In (1) $\mathrm{H}^{\mathrm{SC}}$ stands for the total angular momentum of the satellite system with respect to the mass center $\mathrm{C}$ based on a inertia frame. $\mathrm{H}^{\mathrm{B} / \mathrm{C}}$ stands for the angular 
momentum of the satellite without the reaction wheels. $\mathbf{H}^{\text {WiC }}$ stands for the angular momentum of the reactions wheels. Thus,

$$
\mathbf{H}^{W, / C}=\mathbf{H}^{W_{i} / C_{i}}+m_{W_{i}}\left(\mathbf{r}_{W_{i}} \times \dot{\mathbf{r}}_{w_{i}}\right) \text {. }
$$

The angular momentum of the system is

$$
\mathbf{H}^{S / C}=\mathbf{H}^{B / C}+\sum_{i=1}^{3} \mathbf{H}^{W_{i} / C_{i}}+\sum_{i=1 .}^{3} m_{w_{i}}\left(\mathbf{r}_{\tilde{w}_{i}} \times \dot{\mathbf{r}}_{\boldsymbol{w}_{i}}\right)
$$

or in terms of angular velocities,

$$
\mathbf{H}^{S / C}=\mathbf{I}^{B / C} \omega^{B}+\sum_{i=1}^{3} \mathbf{I}^{F_{i} / C} \omega^{W_{i}}+\sum_{i=1}^{3} m_{w_{i}}\left(\mathbf{r}_{r_{i j}} \times \dot{\boldsymbol{r}}_{w_{i}}\right),
$$

where $\mathrm{I}^{\mathrm{W} v \mathrm{Ci}}$ and $\mathrm{I}^{\mathrm{B} / \mathrm{C}}$ are $3 \times 3$ inertia matrices of $\mathrm{W}_{\mathrm{i}}$ about the mass center $\mathrm{C}_{\mathrm{i}}$ of $\mathrm{W}_{\mathrm{i}}$ and the system $B$. Consider each reaction wheel has spin angular velocity $\Omega_{\mathrm{i}}$ along it axis $d_{i}$, we have

$$
\begin{aligned}
& \omega^{W_{i}}=\omega^{B}+\Omega_{i} \mathbf{d}_{i} \text {. Thus, } \\
& \mathbf{H}^{s i c}=\mathbf{I}^{B / \varepsilon} \omega^{2}+\sum_{i=1}^{3} \mathbf{I}^{w_{i} / \varepsilon}\left(\omega^{3}+\Omega_{i} \mathbf{d}_{i}\right)+\sum_{i=1}^{3} m_{x_{i}}\left(\mathbf{r}_{w_{i}} \times \dot{\mathbf{r}}_{w_{i}}\right) \\
& =\mathbf{I}^{\beta / c} \omega^{\beta}+\sum_{i=1}^{3} \mathbf{I}^{w_{i} / c_{i}}\left(\omega^{8}+\Omega_{i} \mathbf{d}_{i}\right)+\sum_{i=1}^{3} m_{\psi_{i}}\left(\mathbf{r}_{w_{i}} \times\left(\omega^{8} \times \mathbf{r}_{w_{i}}\right)\right) \\
& =\mathbf{I}^{s / C} \omega^{3}+\sum_{i=1}^{3} \mathbf{I}^{m / c}\left(\omega^{3}+\Omega_{i} \mathbf{d}_{i}\right)+\sum_{i=1}^{3} m_{i+i}\left[\begin{array}{ccc}
y_{i}^{2}+z_{i}^{2} & -x_{i} y_{i} & -x_{i} z_{i} \\
-x_{i} y_{i} & x_{i}^{2}+z_{i}^{2} & -y_{i} z_{i} \\
-x_{i} z_{i} & -y_{i} z_{i} & x_{i}^{2}+y_{i}^{2}
\end{array}\right] \omega^{3}
\end{aligned}
$$

In equation (6) $x_{i}, y_{i}$, and $z_{i}$ are the coordinates of $\mathbf{r}_{i}$. Thus,

$$
\begin{aligned}
& \mathbf{H}^{S / C}=\mathbf{I}^{*} \omega^{B}+\mathbf{H}^{*} \text {, where } \\
& \mathbf{I}^{-}=\mathbf{I}^{B / C}+\sum_{i=1}^{3} \mathbf{I}_{i}^{w_{i} / C_{1}}+\sum_{i=1}^{3} m_{w_{i}}\left[\begin{array}{ccc}
y_{i}^{2}+z_{i}{ }^{2} & -x_{i} y_{i} & -x_{i} z_{i} \\
-x_{i} y_{i} & x_{i}{ }^{2}+z_{i}^{2} & -y_{i} z_{i} \\
-x_{i} z_{i} & -y_{i} z_{i} & x_{i}^{2}+y_{i}{ }^{2}
\end{array}\right]
\end{aligned}
$$

and $\mathbf{H}^{*}=\sum_{i=1}^{3} \mathbf{l}^{\pi / C} \mathrm{~d}_{t} \Omega_{i}$. If $\mathbf{d}_{i}$ 's are in the principal directions, then $\mathbf{H}^{*}=\mathbf{A} \Omega$.

Setting the time derivative to obtain the dynamic equation of the satellite system. One obtain

$$
\mathbf{N}=\mathbf{I}^{*} \dot{\omega}^{B}+\omega^{8} \times \mathbf{I}^{*} \omega^{B}+\omega^{8} \times \mathbf{A} \Omega+\mathbf{A} \dot{\Omega},
$$

The angular acceleration of the reaction wheels $\Omega$ can be used as the control input to the system.

\section{Fuzzy Cell-Mapping Method for The Attitude Maneuvering Control}

In this study, we follow the cell-mapping procedure to solve for the optimal control table for the $\sigma^{\text {th }}$ order satellite maneurering and stabilization problem. Because the system is very complicated, it is desirable to use a simple algorithm for the defuzzifying process. Referring to figure 2 , the control effort $\hat{T}_{\mathrm{c}}$ is deternined by 


$$
\hat{T}_{c}=\frac{1}{6} \sum_{i=1}^{0}\left[\frac{d_{12}}{h_{i}} \hat{T}\left(Z_{0}\right)+\frac{d_{11}}{h_{i}} \hat{T}\left(Z_{i}\right)\right]
$$

where $\hat{T}=\left[\begin{array}{lll}T_{w 1} & T_{w 2} & T_{w 3}\end{array}\right]^{T}$. and $h_{i}$ are the sizes of the cells. The time period for application is

$$
\tau_{c}=\frac{1}{6} \sum_{i=1}^{\delta}\left[\frac{d_{i 2}}{h_{i}} \tau\left(Z_{0}\right)+\frac{d_{i 1}}{h_{i}} \tau\left(Z_{i}\right)\right]
$$

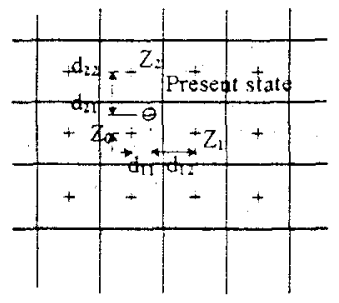

Figure 2 The Cell Structure and The Fuzzy Interpolation

\section{Simulation Results}

The simulation example follows the parameters proposed for the ROCSAT-1 satellite. Consider the Euler axis/angle, we use 17 cells in the finest dimension, and for some dimensions we only use 5 cells. The time of action thus must be long enough so that the system can be driven into a different cell. The partition of the cell is listed below

\begin{tabular}{|c|c|c|c|c|}
\hline state & range & No. of cells & cell sizes & target cell \\
\hline$x_{1}$ & $0 \sim \pi$ & 9 & $0.3+91$ & 1 \\
\hline$x_{2}$ & $0 \sim \pi$ & 9 & 0.3491 & $1 \sim 9$ \\
\hline$x_{3}$ & $-\pi \sim \pi$ & 17 & 0.3696 & $1 \sim 17$ \\
\hline$x_{4}$ & $-0.075 \sim 0.075$ & 5 & 0.03 & 3 \\
\hline$x_{5}$ & $-0.075 \sim 0.075$ & 5 & 0.03 & 3 \\
\hline$x_{6}$ & $-0.075 \sim 0.075$ & 5 & 0.03 & 3 \\
\hline
\end{tabular}

Accordingly, the partition for the forces are set to 0, and $\pm 0.38 \mathrm{~V}-\mathrm{m}$ in all three axes, and the unit for the reaction time is set to $0.75 \mathrm{sec}$.

Figures $3 \mathrm{a}-\mathrm{d}$ shows a maneurering process starting from $\phi=\pi, \psi=\pi$ and $\theta=\pi$ to reach the origin. Figure $3 \mathrm{a}$ and $3 \mathrm{~b}$ show the variation of the rotation angles and the Euler angles, respectively. Figure $3 c$ shows the angular relocities, and figure $3 d$ shows the input torque. The satellite accelerates in all three axes toward the target attitude. The inputs then reduce to zero as the angular reaches a pre-specified limit. The system will then decelerate to settle on the target attitude. From figure $3 \mathrm{~d}$, one can see that the entire system follows a bang-zero-bang control strategy, and the rotational angles move along the optimal trajectories toward the origin. Figures ta$d$ shows the corresponding results with crisp cell-mapping control. It is clear that 
the control effort are not adjusted according to the exact location of the system state. The resulting control shows a overshoot. This is also a common phenomenon in the cell-mapping control.

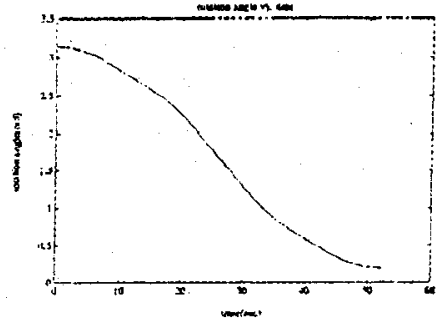

Figure 3a Satellite Rotation Angle (Fuzzy Cell-Mapping)

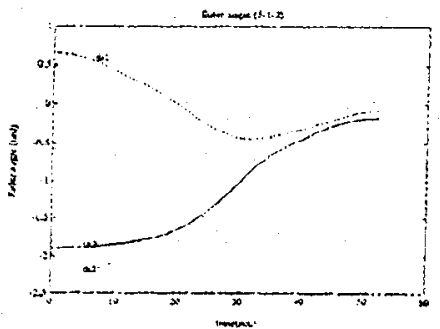

Figure 3b Satellite Euler Angle

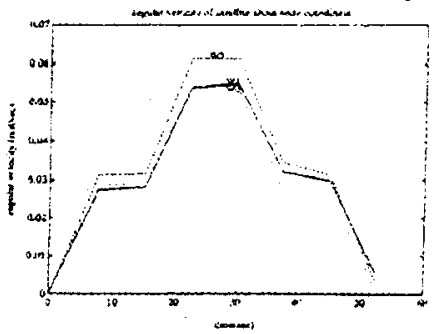

Figure 3c Angular Velocity

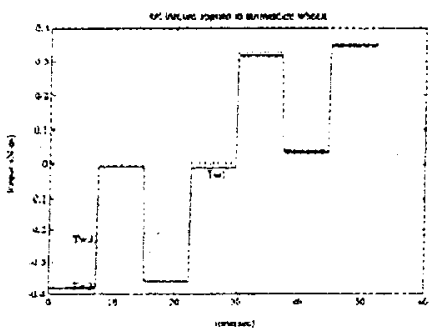

Figure 3d Input Torque Variation

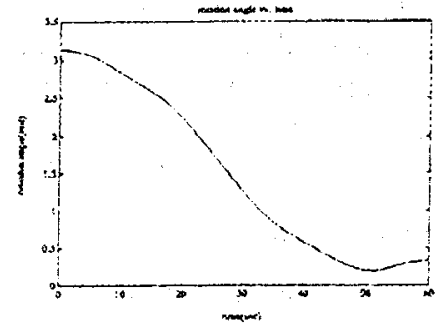

Figure 4a Satellite Rotation Angle (Crisp Cell-Mapping)

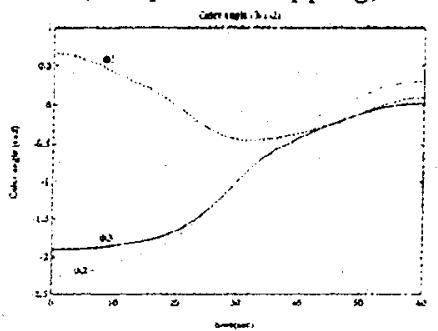

Figure tb Satellite Euler Angle

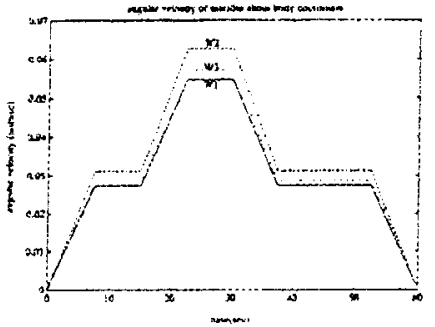

Figure to Angular Velocity

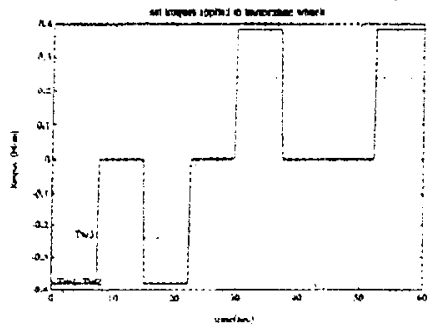

Figure $4 d$ Input Torque Variation 


\section{Conclusions}

A fuzzy cell-mapping control is applied to the satellite attitude maneuvering and stabilizing control. The method includes both the strong capability of the cellmapping method to treat the complex satellite attitude dynamic problem and the smooth control from the fuzzy controller. Simulation results show that the method achicve both maneuvering and stabilization with a smooth transition between the control actions. The control still follows closely the optimal control performance.

\section{Acknowledgments}

The project is supported by National Science Council, Taiwan R.O.C. under project No. NSC-83-NSPO-B-RDD-002-04C.

\section{REFERENCES}

Bois, E. and Kovalevsky, J.. 1990, "Analytical model of the rotation of an artificial satellite," Journal of Guidance. Vol.13, No.6, July-Aug., pp.638-643.

Brun, K-W and Wie, B., 1991, "Robust $H_{x}$ control sign or the space station with structured parameter uncertainty," Journal of Guidance, Vol.14, No.6, Nov.-Dec., pp. $1115-1122$.

Dahl, P.R., 1971, "A twin wheel momentum bias/reaction jet spacecraft control system," ALA4 Paper 71-951, Aug.

Dougherty, H.J.. Lebsock. K.L., and Rodden. J.J.. 1971. "Attitude stabilization of synchronous communications satellites employing narrow-beam antennas," Journal of Sapcecraft and Rockets. Vol.8. Aut., pp.834-841.

Hale, A.L.. Lisowski, R.J., and Dahl, W.E. 1985. "Optimal simultaneous structural and control design of maneurering flexible spacecraft," Journal of Guidance, Vol.8. No.1. Jan.-Feb. pp.86-93.

Hughes. P.C. 1986, Spacecraft attitude dynamics, John Wiley \& Sons. New York. Chichester, Brisbane. Toronto. Singapore.

Hsu. C.S., 1980). "A theon of cell-to-cell mapping dynamical systems", Journal of Applied Mechanics Trans of ASME. Vol +7. Dec pp.931-939.

Hsu, C.S. 1985. "A discrete method of optimal control based upon the cell state space concept". Journal of optimization theory and applicatons, Vol.t6, No.4, Aug., pp. 5+7-569.

Hsu, C.S. 1987. Cell-to-cell mapping. Springer-Verlag.

Wertz, J.R., 1978. Spacecraft attitude determination and control, Edited, Kluwer Academic Publishers. Dordrecht. Boston. London.

Wie. B. Lehner. J.A. and Plescia. C.T. 1985, "Roll/yaw control of a flexible spacecrift using skewed bias momentum wheels." Journal of Guidance. Vol.8, No.t. July-Aug.. pp. $4+7-453$

Yen. J.-Y.. Chao. W.-C.. and Lu. S.-S.. 1994. "A fuzzy cell mapping method for a time sub-optimal control implementation." Control Engineering Practice, Vol.2, No.2. pp. 247-254. 